\title{
On the dynamic response of an ocean to wind stress in an equatorial region-ii: further exact solutions of the rossby equation
}

\author{
M. Z. Rahman, A. B. M. Shamim. Ul Hasan \\ Department of Applied Mathematics, University of Rajshahi, Rajshahi-6205, Bangladesh \\ Email address: \\ mzillurr@yahoo.com(M. Z. Rahman), mshamim.appm.ru@gmail.com(A.B.M. S. U. Hasan)
}

\section{To cite this article:}

M. Z. Rahman, A. B. M. Shamim.U1 Hasan. On the Dynamic Response of an Ocean to Wind Stress in an Equatorial Region-II: Further Exact Solutions of the Rossby Equation. Pure and Applied Mathematics Journal. Vol. 2, No. 3, 2013, pp. 110-118.

doi: $10.11648 /$ j.pamj.20130203.11

\begin{abstract}
In a beforehand paper they found some exact and explicit solutions for the standard Rossby form of the equation for the stream function for some specified and realistic wind stress. This equation, which is a third order linear partial differential equation for the stream function, relates the rate of change of vertical vortices to the curl of the applied wind stress. The equation involves the gradient of the Coriolis parameter and has particular relevance to the equatorial region, such as the North Indian Ocean. Some interesting physical properties of the solutions are considered. In this paper we find some more complicated but similar exact and explicit solutions. Some properties for these solutions are derived which are in some sense complementary to the kind of properties of the simpler solutions considered in advance.
\end{abstract}

Keywords: Rossby Equation, Velocity, Coriolis Parameter, Gradient

\section{Introduction}

Some years ago the investigation [7] made a detailed study of the dynamic response of the North Indian Ocean to the onset of the Southwest Monsoon. The mathematical problem is fairly complicated since, even with the simplifying assumption of uniform depth and neglect of variation of velocity wit depth, one gets a system of nonlinear partial differential equations, which are intractable. The linearized theory, however gives reasonable results, as [8] shows. One of these results is the connection between the onset of the Southwest Monsoon and the relatively strong Somali current that occurs on the east coast of Africa about a month later. There have been other similar studies such that of investigation [1], [7], [8].

In the investigation [5] they found some exact and explicit solutions for the standard Rossby form of the equation for the stream function for some realistic given wind stress; this is a third order partial differential equation [5]. Some properties of the solution were derived. In this paper we extend these solutions to similar but more complicated exact and explicit solutions. We consider further properties of the solutions which are in a sense complementary to the ones derived for the simpler solutions considered earlier. In particular, we determine the streamlines, which turn out to be ellipses with a realistic global structure, with the velocity vanishing near the centre, reaching a maximum as one move away from the centre, and again vanishing at great distance from the centre, reminiscent of cyclones. As before [5] they find two kinds of solutions, the first having Gaussian factors with exponential 'tails' the second being bounded that is, vanishing strictly outside a bounded region in space and time.

As indicated in paper [5] although the Rossby form itself is approximate and the linearity is a restriction, never the less the exact and explicit form of the solutions and in particular the unusual nature of the solutions with bounded support (we are not aware of any such solutions in this context), may help to provide some insights into this important problem and related ones [3]. We derive the further exact solutions of Gaussian form in $\$ 2$, and consider some properties in $\S 3$. The further, explicit exact solution with bounded support and some properties are considered in $\S 4$. 


\section{Further Exact Solutions of Gaussian Form}

We have the Rossby form of equation as used in paper [5].

$$
\psi_{t c x}+\psi_{t y y}+\beta \psi_{x}=G_{x}-F_{y}
$$

Where $\psi$ is steam function, the usual notations are used the paper [5].

Let

$$
\begin{gathered}
Q=Q_{0} Q_{1} Q_{2}, \\
Q_{0}=\exp \left(-1 / 2 \alpha t^{2}\right) \\
Q_{1}=\exp \left(-1 / 2 \gamma x^{2}\right) \\
Q_{2}=\exp \left(-1 / 2 \gamma^{\prime} y^{2}\right),
\end{gathered}
$$$$
\bar{\psi}_{t c x x}+\bar{\psi}_{t y y}+\beta \bar{\psi}_{x}-2 \gamma x \bar{\psi}_{t x}-\alpha t \bar{\psi}_{x x}+2 \alpha y t x \bar{\psi}_{x}+\left(-\gamma+\gamma^{2} x^{2}\right) \bar{\psi}_{t}-\alpha t\left(\left(-\gamma+\gamma^{2} x^{2}\right) \bar{\Psi}-\right.
$$$$
\beta \gamma x \bar{\psi}-2 \gamma^{\prime} y \bar{\psi}_{t y}+\left(-\gamma^{\prime}+\gamma^{2} y^{2}\right) \bar{\psi}_{t}-\alpha t \bar{\psi}_{y y}+2 \alpha \gamma^{\prime} t y \bar{\psi}_{y}-\alpha t\left(-\gamma^{\prime}+\gamma^{\prime} y^{2}\right) \bar{\psi}=\bar{G}_{x}-\gamma x \bar{G}-\bar{F}_{y}+\gamma^{\prime} y \bar{F}
$$

We now assume $\psi$ to have the following form:

$$
\bar{\psi}=t\left(a x^{2}+b y^{2}+c\right)
$$

Where $\mathrm{a}, \mathrm{b}, \mathrm{c}$ are constants. Then the following derivatives are obtained readily from (2.6):

$$
\left.\begin{array}{lc}
\bar{\psi}_{t x x}=2 a, & \bar{\psi}_{t y y}=2 b \\
\bar{\psi}_{t}=a x^{2}+b y^{2}+c \bar{\psi} t x=2 a x & \bar{\psi}_{x}=2 a t x, \\
\bar{\psi}_{t y}=2 b y, & \bar{\psi}_{y y}=2 b t, \quad \bar{\psi}_{y}=2 b t y .
\end{array}\right\}
$$

Next assume $\bar{F}, \bar{G}$, to be of the following form (the reason for the choice of $\bar{\psi}, \bar{F}, \bar{G}$ will be explained later), where the a's and b's are constants:

$$
\begin{gathered}
\bar{G}=t^{2}\left(a^{\prime} x^{\prime}+\bar{a} x\right)+t\left(\hat{a} x^{2}+\hat{b} y^{2}\right)+a^{\prime} x^{3}+\bar{b}^{\prime} x y^{2}+\overline{\bar{a}} x \\
\bar{F}=t^{2}\left(a^{\prime \prime} x^{2} y+b^{\prime \prime} y^{3}+\bar{b} y\right)+\hat{c} t x y+\bar{a}^{\prime} x^{2} y+\bar{b}^{\prime \prime} x y 3+\overline{\bar{b}} y .
\end{gathered}
$$

Obtaining the derivatives $\bar{G}_{x}, \bar{F} y$, and substituting these and all the derivatives of $\bar{\psi}$ into (2.5) finally we get.

$$
\begin{aligned}
& 2(a+b)+2 a \beta t x-4 a \gamma x^{2}-2 a \alpha t^{2}+4 a \alpha \gamma t^{2} x^{2}+\left(-\gamma+\gamma^{2} x^{2}\right)\left(a x^{2}+b y^{2}+c\right)-\alpha t^{2}\left(-\gamma+\gamma^{2} x^{2}\right) \\
& \left(a x^{2}+b y^{2}+c\right)-\beta \gamma t x\left(a x^{2}+b y^{2}+c\right)-4 b \gamma^{\prime} y^{2}+\left(-\gamma^{\prime}+\gamma^{2} y^{2}\right)\left(a x^{2}+b y^{2}+c\right)-2 b \alpha t^{2}+4 b \alpha \gamma^{\prime} t^{2} y^{2}-\alpha t^{2} \\
& \left(-\gamma^{\prime}+\gamma^{\prime 2} y^{2}\right)\left(a x^{2}+b y^{2}+c\right) \\
& =t^{2}\left(3 a^{\prime} x^{2}+\bar{a}\right)+2 \hat{a} t x+3 \bar{a}^{\prime} x^{2}+\bar{b}^{\prime} y^{2}+\overline{\bar{a}}-t^{2}\left(a^{\prime \prime} x^{2}+3 b^{\prime \prime} y^{2}+\bar{b}\right)-\hat{c} t x-\bar{a}^{\prime \prime} x^{2}-3 a \bar{b}^{\prime \prime} y^{2}-\overline{\bar{b}} \\
& -\gamma t^{2}\left(a^{\prime} x^{4}+\bar{a} x^{2}\right)-\gamma t\left(\hat{a} x^{3}+\hat{b} x y^{2}\right)-\gamma\left(\bar{a}^{\prime} x^{4}+\bar{b}^{\prime} x^{2} y^{2}+\overline{\bar{a}} x^{2}\right)+\gamma^{\prime} t^{2}\left(a^{\prime \prime} x^{2} y^{2}+b^{\prime \prime} y^{4}+\bar{b} y^{2}\right) \\
& \hat{c} \gamma^{\prime} t x y^{2}+\bar{a}^{\prime \prime} \gamma^{\prime} x^{2} y^{2}+b^{\prime \prime} y^{4}+\overline{\bar{b}} y^{2}
\end{aligned}
$$


If we now equate the coefficients of various terms on the left and right hand sides of this equation, we find that all the unknown constants appearing on the right hand side that is, all the coefficients appearing in the equations $(2.8 \mathrm{a}$, b) for $\bar{F}$ and $\bar{G}$ can be expressed in terms of the constants appearing on the left hand side, namely, $\beta, \alpha, \gamma^{\prime}, a, b, c$ we will not give details here but state the result and verify a couple of cases. The unknown constants on the right on the right hand side of (2.9) are given as follows:

$$
\left.\begin{array}{l}
\hat{a}=a \beta, \hat{c}=c \beta \gamma, \hat{b}=\left(b+c \gamma^{\prime}\right) \beta \\
a^{\prime}=a \alpha \gamma, \quad \bar{a}^{\prime}=-a \gamma, \overline{b^{\prime}}=c \gamma^{2}, \\
a^{\prime \prime}=-a \alpha \gamma^{\prime}-b \alpha \gamma^{2} / \gamma^{\prime}, b^{\prime \prime}=-b \alpha \gamma^{\prime} \\
\bar{a}^{\prime \prime}=a \gamma^{\prime}+b \gamma^{2} / \gamma^{\prime}+c \gamma^{\prime} \bar{b}^{\prime \prime}=b \gamma^{\prime} \\
\bar{a}=c \alpha \gamma+b \alpha \gamma^{\prime} / \gamma^{\prime}-2 a \alpha, \bar{b}=2 b \alpha+b \alpha \gamma / \gamma^{\prime}-c \alpha \gamma^{\prime}, \\
\overline{\bar{a}}=2 a-c\left(\gamma+\gamma^{\prime}\right)-b \gamma / \gamma^{\prime}, \overline{\bar{b}}=-b \gamma / \gamma^{\prime}-2 b .
\end{array}\right\}
$$

We verify two cases at random. The coefficients of $t^{2} z^{2}$ in (2.9) require the satisfaction of the following equation:

$$
5 a \alpha \gamma-c \alpha \gamma^{2}+a \alpha \gamma^{\prime}=3 a^{\prime}-a^{\prime \prime}-\bar{a} \gamma
$$

Which is readily verified if we substitute on the right hand side for $a, a^{\prime \prime}, \bar{a}$ from (2.10). Next consider of $x^{2} y^{2}$ in (2.10) this yields the following equation:

$$
b \gamma^{2}+a \gamma^{\prime 2}=-\bar{\gamma} b^{\prime}+\gamma^{\prime} \bar{a}^{\prime \prime}
$$

Which is again verified if we substitute for $\bar{b}^{\prime}, \bar{a}^{\prime \prime}$ from (2. 10)

\section{Some Properties of the Solution}

The horizontal components $(\mathrm{u}, \mathrm{v})$ of the velocity are given by

$$
u=-\psi_{y,} \quad v=\psi_{x}
$$

For the form (2.6) of the function $\psi$ we get

$$
\begin{aligned}
& u=t y\left[-2 b+\gamma^{\prime}\left(a x^{2}+b y^{2}+c\right)\right] Q \\
& v=t x\left[2 a-\gamma\left(a x^{2}+b y^{2}+c\right)\right] Q
\end{aligned}
$$

It is easier to discuss specific properties of the solution in a slightly less general and simpler form than those given in (3.2a, b) and the forms of F, G given in (2.3) and (2.8a, b). The form of (3. 2a, b) suggests two limiting case, as follows:

$$
\begin{gathered}
\text { Case (A): } 2 \mathrm{~b}=\gamma^{\prime} c, 2 a=\gamma c . \\
\text { Case (B) } \mathrm{a}=\mathrm{b}=0 .
\end{gathered}
$$

We study these cases in some detail.

Case (A). In this case the velocity components are as follows:

$$
\begin{aligned}
& u=1 / 2 c \gamma^{\prime} t y\left(\gamma x^{2}+\gamma^{\prime} y^{2}\right) Q \\
& v=-1 / 2 c \gamma t x\left(\gamma x^{2}+\gamma^{\prime} y^{\prime}\right) Q
\end{aligned}
$$

From (3. 3a, b) we get

$$
\gamma x u+\gamma^{\prime} y v=0
$$

This relation shows that the velocity vector $(\mathrm{u}, \mathrm{v})$ is perpendicular to the vector $\left(\gamma x, \gamma^{\prime} y\right)$. Consider the quadratic function $\mathrm{E}(\mathrm{x}, \mathrm{y})$ defined as follows so that the curves $\mathrm{E}(\mathrm{x}, \mathrm{y})=$ constant are ellipses:

$$
E(x, y)=1 / 2\left(\gamma x^{2}+\gamma^{\prime} y^{2}\right)=\text { Constant }
$$

Clearly, the normal to such an ellipse at the point (x,y) has direction proportional to the vector (Ex, Ey), that is to the gradient of $\mathrm{E}$. We have

$$
(\mathrm{Ex}, \mathrm{Ey})=\left(\gamma x, \gamma^{\prime} y\right)
$$

Since, from (3.4) the velocity vector $(\mathrm{u}, \mathrm{v})$ is normal to the vector (3.6), and since the latter is normal to the ellipse (3.5), it follows that the velocity vector is along the ellipse; in other words, the streamlines are the concentric ellipses given by (3.5) for any give fixed value of the time t. Further for any given $y>0$ (that is, position north of the equator), $\mathrm{u}<0$ for $\mathrm{t}<0$ and $\mathrm{u}>\mathrm{o}$ for $\mathrm{t}>\mathrm{o}$ (assuming $\mathrm{c}>0$ ), while for $\mathrm{y}<0$, $\mathrm{u}>$ for $\mathrm{t}<0$ and $\mathrm{u}<0$ for $\mathrm{t}>0$. One can give similar analysis of the v-component of the velocity. All this behavior is summarized in Figs. (1, 2, 3 and 4) where the signs of the velocity components are indicated at the four points $\left(x_{0}, y_{0}\right),\left(x_{0}-y_{0}\right),\left(-x_{0}, y_{0}\right),\left(-x_{0}-y_{0}\right)$ for $\mathrm{t}>0$ and $\mathrm{t}<0$ and typical streamlines are also indicated for $\mathrm{t}>0$ and $\mathrm{t}<0$ in figs. 1 and 2 are quite consistent with the streamlines shown in Figs 3 and 4. It is worth noting that the semi axes of the ellipses (3.5) are proportional to $1 / \sqrt{\gamma}$ and $1 \sqrt{\gamma^{\prime}}$, which are related to the Gaussian 'spread' of the disturbance, as indicated earlier.

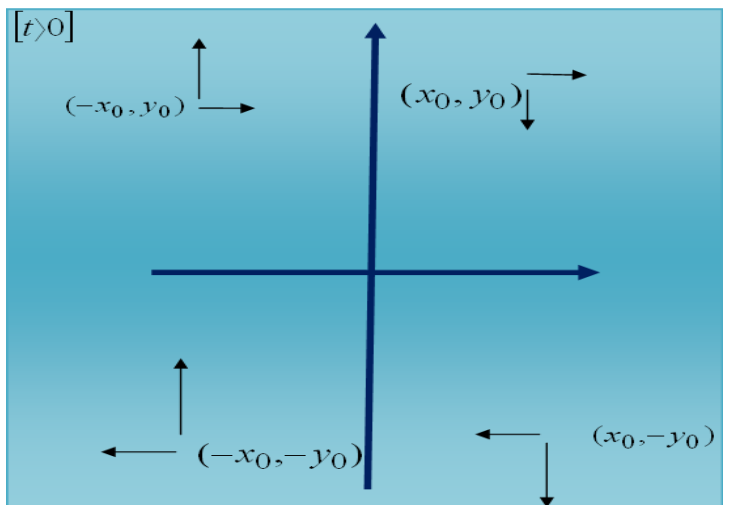

Figure 1. This diagram indicates the signs of the velocity components $(u, v)$ at the four points shown for $t>0$ for the solution $(3.3 a, b)$. 


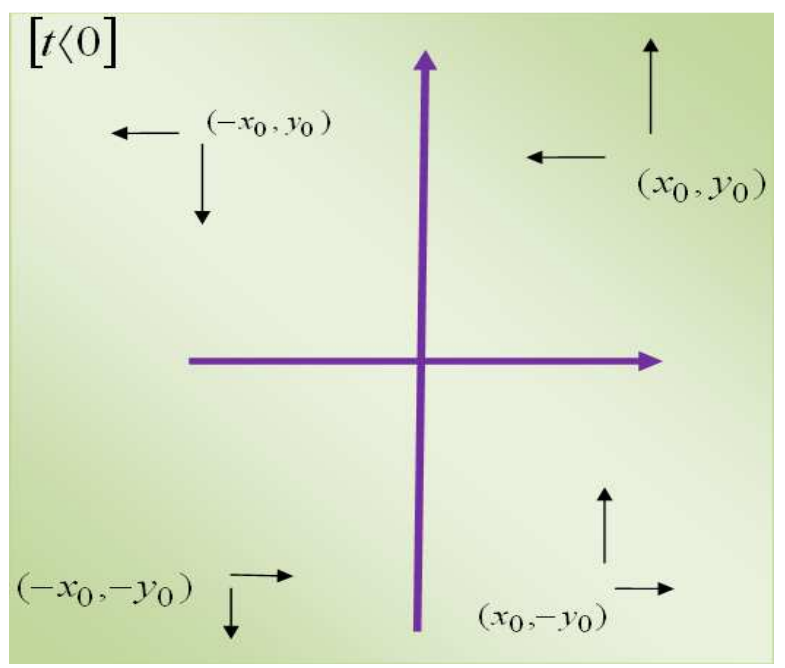

Figure2. This diagram is similar to that of Fig.1, but for $t<0$.

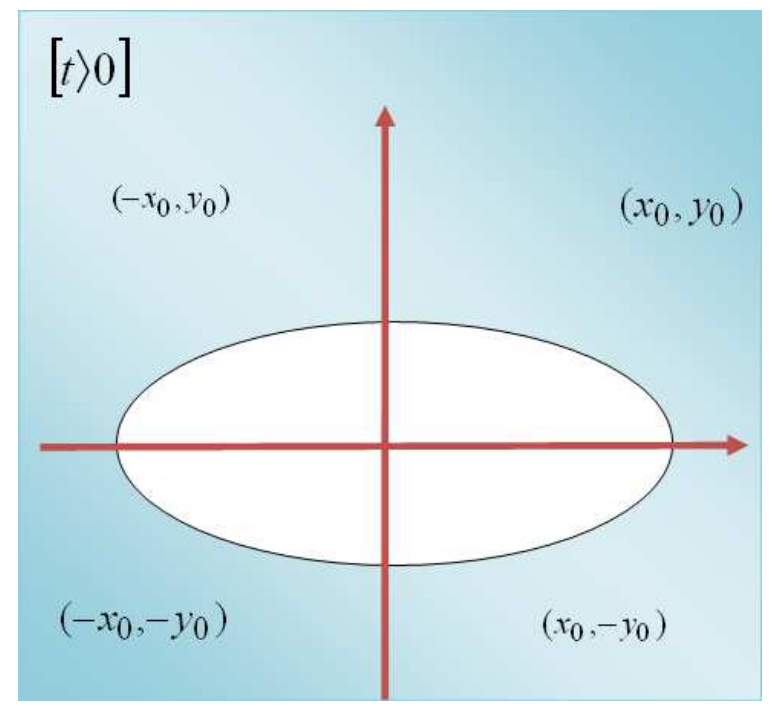

Figure 3. This diagram gives the elliptical streamline corresponding to that of Fig. 1. The directions of the velocity shown at the four points in Fig. 1 are consistent with the directions of the streamline at the same points here. Other streamlines (for a fixed value of $t$ ) are concentric ellipses. $t<0$

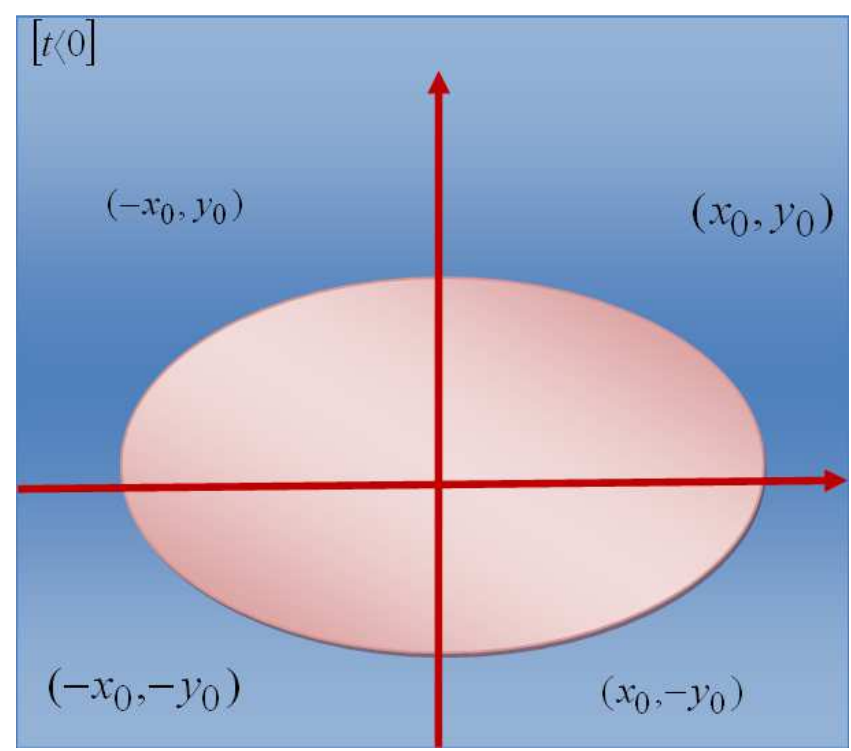

Figure.4. This diagram corresponds to that of Fig. 2 in the same way that the diagram of Fig. 3 corresponds to that Fig. 1.

The coefficient of the expressions $\bar{G}, \bar{F}$ occurring in (2. 10 ) in this case reduce to the following (we are continuing with case (A) :

$$
\left.\begin{array}{l}
\hat{a}=1 / 2 c \beta \gamma, \hat{c}=c \beta \gamma, \hat{b}=(3 / 2) c \beta \gamma^{\prime}, \\
a^{\prime}=1 / 2 c \alpha \gamma^{2}, a^{\prime}=-1 / 2 c \gamma^{2}, \bar{b}^{\prime}=c \gamma^{2}, \\
a^{\prime \prime}=-1 / 2 c \alpha \gamma\left(\gamma+\gamma^{\prime}\right), b^{\prime \prime}=-1 / 2 c \alpha \gamma^{\prime 2} \\
\bar{a}^{\prime \prime}=-1 / 2 c \gamma\left(\gamma+3 \gamma^{\prime}\right), b^{\prime \prime}=-1 / 2 c \gamma^{\prime 2}, \\
\bar{a}=-1 / 2 c \alpha \gamma=\bar{b}, \\
\overline{\bar{a}}=-c\left(1 / 2 \gamma+\gamma^{\prime}\right)=\overline{\bar{b}} .
\end{array}\right\}
$$

The corresponding expressions for $\bar{G}, \bar{F}$ are as follows:

$$
\begin{gathered}
\bar{G}=1 / 2 c \alpha \gamma(\gamma x+1) x t^{2}+1 / 2 c \beta\left(\gamma x^{2}+3 \gamma^{\prime} y^{2}\right) t+c x\left(-1 / 2 \gamma^{2} x^{2}+\gamma^{2} y^{2}-\gamma^{\prime}-1 / 2 \gamma\right) \\
\bar{F}=1 / 2 c \alpha\left[-\gamma\left(\gamma+\gamma^{\prime}\right) x^{2}-\gamma^{\prime 2} y^{2}+\gamma\right] y t^{2}+c \beta \gamma t x y+c \gamma\left[1 / 2 \gamma\left(\gamma+3 \gamma^{\prime}\right) x^{2}+1 / 2 \gamma^{\prime 2} y^{2}-1 / 2 \gamma-\gamma^{\prime}\right]
\end{gathered}
$$

These forms of $\bar{G}, \bar{F}$ are not very illuminating except to note that it takes fairly complicated forms of wind stress to produce the relatively simple expressions $(3.3 \mathrm{a}, \mathrm{b})$ for the velocity components $(\mathrm{u}, \mathrm{v})$ As will be shown below, the expressions for $(\mathrm{u}, \mathrm{v})$ become even simpler and more transparent. If we restrict further to

$$
\gamma=\gamma^{\prime}
$$

A further point to note is the following. Suppose we have fixed values of $\mathrm{c} \gamma, \gamma^{\prime}, \alpha$. Then the velocity components (u, v) are fixed (for any given $\mathrm{t}, \mathrm{x}, \mathrm{y})$ by (3.3a, b) while the $\bar{G}, \bar{F}$ are given by $(3.8 \mathrm{a}, \mathrm{b})$.
Now the coriolis parameter $\beta$ representing the rotation of the earth occurs only in the expression for $\bar{G}, \bar{F}$. If we set $\beta=0$ in these expressions, this does not appear to have any effect on the components $(\mathrm{u}, \mathrm{v})$ given by $(3.3 \mathrm{a}, \mathrm{b})$. This situation (with $\beta=0$ ) represents an infinite flat 'sea' in which currents are produced by the given wind stress. The interpretation here is that when $\beta \neq 0$ (that is when we have rotation), then it requires the corresponding modified stresses $\bar{G}, \bar{F}$ (with factor Q), to produce the same velocity field (u, v) of currents.

Still continuing with Case (A), we set $\gamma=\gamma^{\prime}$, that is, we 
consider the Gaussian 'spread' in the $\mathrm{x}$ and $\mathrm{y}$ directions to be the same. It is readily seen from $(3.3 a, b)$ that in this in this case the square of the magnitude of the velocity is as follows:

$$
u^{2}+v^{2}=(1 / 4) c^{2} \gamma^{4} t^{2} r^{6} \exp \left(-\alpha t^{2}\right) \exp \left(-\gamma r^{2}\right),
$$

Where we have set $\mathrm{r}=\mathrm{x}+\mathrm{y}$ and written out the exponentials explicitly. Note that for any fixed $t$, the streamlines are circles with the origin as centre, reminiscent of cyclones, while the velocity tends to zero both at infinity, being strongly damped by the Gaussian factor as well as at zero, so that the centre of the 'storm' is relatively calm, similar to some actual situations. For any fixed $t$ the maximum of the velocity is the same as the maximum of the square of the velocity. This is seen, by differentiation with respect to $r$, to occur at the following value

$$
\begin{aligned}
r=(3 / \gamma)^{1 / 2} & =r_{0 .} \\
f^{\prime}(r) & =A r^{2}\left(3-\gamma r^{2}\right) \exp \left(-1 / 2 \gamma r^{2}\right), f^{\prime \prime}(r)=A r\left(6-7 \gamma r+\gamma^{2} r^{2}\right) \exp \left(-1 / 2 \gamma r^{2}\right) .
\end{aligned}
$$

The magnitude of the velocity (the velocity being cross radial) for a fixed $\mathrm{t}$ as one moves from $\mathrm{r}=0$ along a straight line in any direction is depicted in Fig. 5, in which $r_{0}$ is the value of $\mathrm{r}$ at which the maximum occurs given by (3.10), while $r_{1}$ and $r_{2}$ are the values at which the given curve changes from being convex towards the r-axis to being concave towards it and vice versa. These two points are obtained by setting equal to zero the second derivative with respect to $r$ of magnitude of the velocity, for fixed $t$. From (3.9) we see that the $r$ dependence of the magnitude of the velocity can be taken as follows.

$$
\left(u^{2}+v^{2}\right)^{1 / 2}=A r^{3} \exp \left(-1 / 2 \gamma r^{2}\right)=f(r),
$$

Where $\mathrm{A}$ is a function of $\mathrm{t}$ that can be read off from (3.9). The first and second derivatives of $\mathrm{f}(\mathrm{r})$ with respect to $\mathrm{r}$ are as follows:

Thus $f^{\prime}(r)=0$ gives the value $r=r_{0}$ given by (3.10) while $f^{\prime \prime}(r)=0$ yield the values $r_{1}, r_{2}$ as follows:

$$
r=(1 / \gamma)^{1 / 2}=r_{1}, r=(6 / \gamma)^{1 / 2}=r_{2}
$$

As expected $r_{1}<r_{0}<r_{2}$. One can carry out a similar analysis with respect to $\mathrm{t}$ for fixed $\mathrm{r}$. Indeed the magnitude of the velocity in this case is

$$
\left(u^{2}+v^{2}\right)^{1 / 2}=B t \exp \left(-1 / 2 \alpha t^{2}\right)=h(t)
$$

Where $B$ is a function of $r$ which can be determined from (3.9) the nature of the t-dependence of the velocity for fixed $r$ is indicated by the first and second derivatives of $h(t)$ with respect to $t$, as follows:

$$
\dot{h}(t)=B\left(1-\alpha t^{2}\right) \exp \left(-1 / 2 \alpha t^{2}\right), \ddot{h}(t)=B \alpha t\left(-3+\alpha t^{2}\right) \exp \left(-1 / 2 \alpha t^{2}\right)
$$

The equation $\mathrm{h}(\mathrm{t})=0$ gives the maximum (for fixed $\mathrm{r}$ ) at $t=t_{0}$, while in the case unlike the function $\mathrm{f}(\mathrm{r})$, we get one non-zero value of $t$,

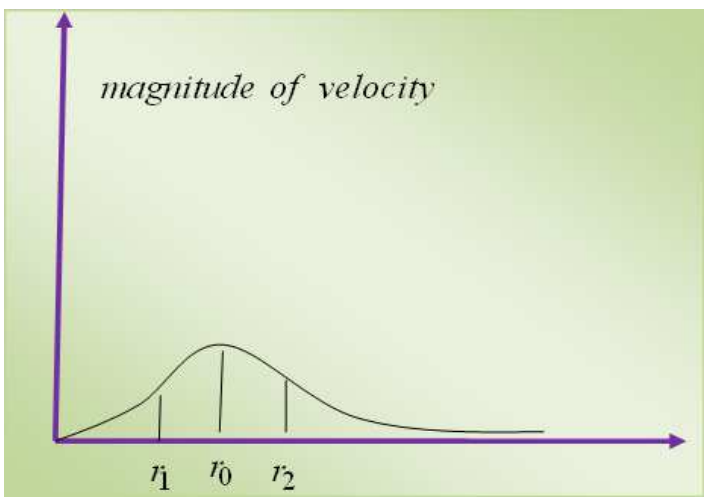

Figure 5. This diagram depicts the behavior of the magnitude of the velocity, Eq. (3.11) as a function of $r$ for fixed the values $r_{0}, r_{1}, r_{2}$ are given by (3.10) (3.13).

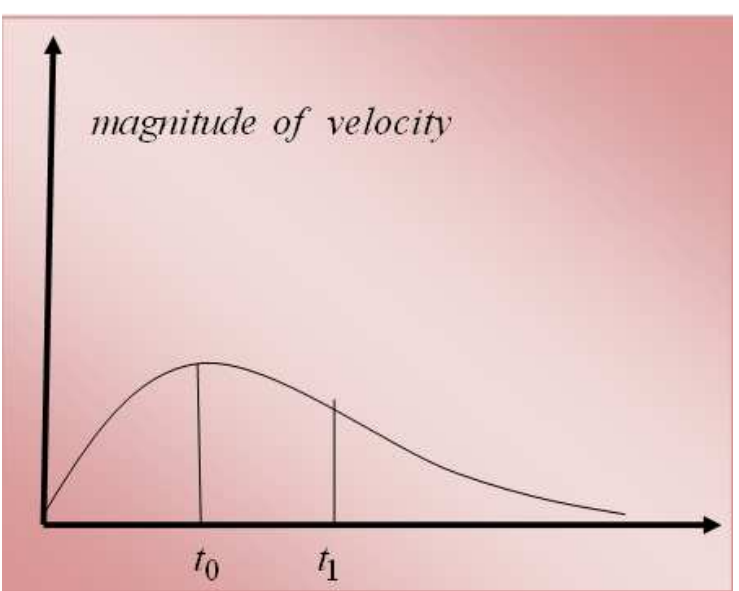

Figure 6. This diagram depicts the depicts the behavior of the magnitude of the velocity, Eq. (3.14), as a function of $t$ for fixed $r$, The values $t_{0}, t_{1}$ are given by (3.16). 


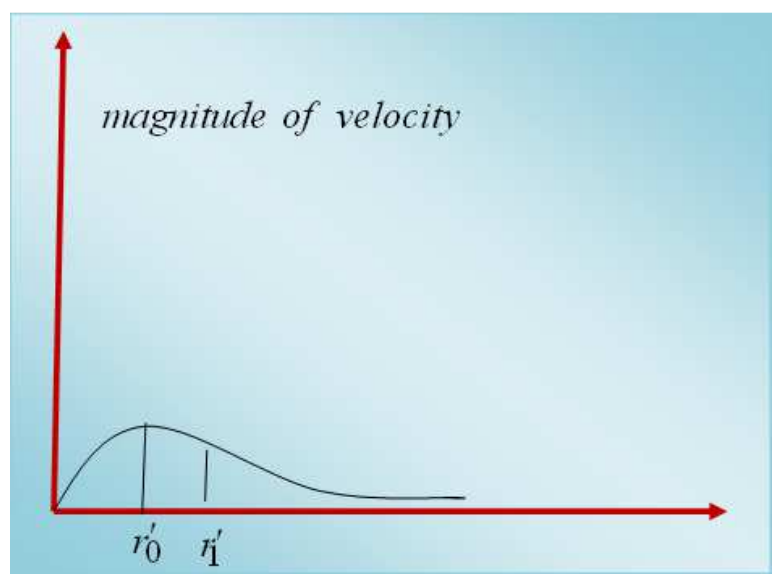

Figure 7. This diagram depicts the behavior of the magnitude of the velocity, Eq. (3.10), as a function of $r$ fixed $t$. The values $r^{\prime}{ }_{0} r^{\prime}{ }_{1}$ are given by (3.19).

Given by $t=t_{1}$, at which point the second derivative vanishes, so that the curve changes from being concave to being convex towards the t-axis; these are as follows:

$$
t_{0}=(1 / \alpha)^{1 / 2}, t_{1}=(3 / \alpha)^{1 / 2}
$$

This behavior is shown in Fig. 6 .

As before the form of the functions $\mathrm{F}, \mathrm{G}$ for $\gamma=\gamma^{\prime}$ is not very illuminating. In particular, the square of the magnitude of the stress, $F^{2}+G^{2}$ is not expressible in terms of $r$ for $\gamma=\gamma^{\prime}$, unlike the expression $u^{2}+v^{2}$.Thus, although the magnitude of the velocity is spherically symmetric, the magnitude of the stress that produces the velocity is not. This is not surprising; it probably arises from the fact that the curl of the vector $(F, G)$ is involved in the determination of the velocity components. This completes case (A).

Case (B).

In this case the velocity components are as follows:

$$
u=c \gamma^{\prime} t y Q, \quad v=-c \gamma t x Q .
$$

As before the velocity components satisfy the equation (3.4), so that the streamlines are again, for a fixed $\mathrm{t}$ concentric ellipses if we again specialize to $\gamma=\gamma^{\prime}$, the magnitude of the velocity is given as follows:

$$
\left(u^{2}+v^{2}\right)^{1 / 2}=c \gamma t r \exp \left(-1 / 2 \alpha t^{2}\right) \exp \left(-1 / 2 \gamma r^{2}\right)
$$

In this case the behavior of the magnitude of the velocity with respect to $r$ for fixed $t$ is the same as that with respect to $\mathrm{t}$ for fixed $\mathrm{r}$, as is clear from (3.18), so that the $\mathrm{r}$ - dependence also has the same form as that depicted in Fig.6. This is shown in fig.7. Thus in this case also we have, for fixed $t$, zero magnitude of velocity at the centre $r=0$, indicating relative calm at the 'centre' of the storm. Note that in Fig. 7 for the r-variable we have the two values $r_{0}^{\prime} r_{1}^{\prime}$ given by (see (3.16)

$$
r_{0}^{\prime}=(1 / \gamma)^{1 / 2}, \quad r_{1}^{\prime}=(3 / \gamma)^{1 / 2}
$$

So that the maximum, at $r_{0}^{\prime}$, occurs at a smaller value than the corresponding maximum $r_{0}$ of case (A) (Fig. 5). This completes the consideration of cases (A) and (B).

\section{Further Exact Solution with Bounded Support}

In this section we consider more solutions of the Rossby equation, rewriting it for convenience:

$$
\psi_{t x x}+\psi_{t y y}+\beta \psi_{x}=G_{x}-F_{y}
$$

And make the following ansatz for $\psi$ :

$$
\begin{array}{ll}
\psi=A \exp \left(B^{2} / S^{\prime}\right), & S^{\prime}<0 \\
=0, & S^{\prime} \geq 0
\end{array}
$$

Where

$$
S^{\prime} \equiv\left(x-x_{0}\right)^{2}+\left(y-y_{0}\right)^{2}-R^{2}
$$

Where $x_{0}=x_{0}(t), y_{0}=y_{0}(t)$ are unspecified function of $\mathrm{t}$, but $\mathrm{R}$ is a constant independent of $\mathrm{t}$. Note that the curves $S^{\prime}=0$ is a circle of radius $\mathrm{R}$ with moving centre $\left(x_{0}(t), y_{0}(t)\right)$.The form (4.2a) is quite distinct to that given by (14) of paper [5] and cannot be reduced to the latter for any functions $x_{0}(t), y_{0}(t)$ and constant $\mathrm{R}$. we proceed to show that the Eq. given by $(4.2 \mathrm{a}, \mathrm{b})$ is an exact solution of (4.1) for certain $F$ and $G$, which will be of the following form

$$
F=\hat{F} \psi, \quad G=\hat{G} \psi
$$

Where $\hat{F}, \hat{G}$ will be determined below

We have

$$
\begin{gathered}
\psi=A \exp \left(\frac{B^{2}}{\left(x-x_{0}\right)^{2}+\left(y-y_{0}\right)^{2}-R^{2}}\right)=A \exp \frac{B^{2}}{S^{\prime}} . \\
\psi_{t}=A \frac{B^{2}}{S^{\prime 2}}\left[2\left(x-x_{0}\right) \dot{x}_{0}+2\left(y-y_{0}\right) \dot{y}_{0}\right] \psi, \psi_{x}=\frac{A B^{2}}{S^{\prime 2}}-\left[2\left(x-x_{0}\right)\right] \psi, \\
\psi_{t x}=\frac{2 A B^{2} \dot{x}_{0}}{S^{\prime 2}} \psi+\frac{8 A B^{2}\left(x-x_{0}\right)^{2} \dot{x}_{0}}{S^{\prime 3}} \psi+\frac{4 A B^{4}\left(x-x_{0}\right)^{2} \dot{x}_{0}}{S^{\prime 4}} \psi .
\end{gathered}
$$




$$
\begin{gathered}
+\frac{8 A B^{2}\left(x-x_{0}\right)\left(y-y_{0}\right) \dot{y}_{0}}{S^{\prime 3}} \psi+\frac{4 A B^{4}\left(x-x_{0}\right)\left(y-y_{0}\right) \dot{y}_{0}}{S^{\prime 4}} \psi \\
\psi_{t x x}=\frac{24 A B^{2}}{S^{\prime 3}}\left(x-x_{0}\right) \dot{x}_{0}-\frac{48 A B^{2}}{S^{\prime 4}}\left(x-x_{0}\right)^{3} \dot{x}_{0}+\frac{12 A B^{4}}{S^{\prime 4}}\left(x-x_{0}\right) \dot{x}_{0} . \\
-\frac{48 A B^{4}}{S^{\prime 5}}\left(x-x_{0}\right)^{3} \dot{x}_{0}-\frac{8 A B^{6}}{S^{\prime 6}}\left(x-x_{0}\right)^{3} \dot{x}_{0}-\frac{48 A B^{4}}{S^{\prime 5}}\left(x-x_{0}\right)^{2}\left(y-y_{0}\right) \dot{y}_{0} . \\
-\frac{8 A B^{6}}{S^{\prime 5}}\left(x-x_{0}\right)^{2}\left(y-y_{0}\right) \dot{y}_{0}+\frac{8 A B^{2}}{S^{\prime 3}}\left(y-y_{0}\right) \dot{y}_{0}+\frac{4 A B^{4}}{S^{5}}\left(y-y_{0}\right) \dot{y}_{0} . \\
\frac{48 A B^{2}}{S^{\prime 4}}\left(x-x_{0}\right)^{2}\left(y-y_{0}\right) \dot{y}_{0}, \\
\psi_{t y y}=\frac{24 A B^{2}}{S^{\prime 3}}\left(y-y_{0}\right) \dot{y}_{0}-\frac{48 A B^{2}}{S^{\prime 4}}\left(y-y_{0}\right) \dot{y}+\frac{12 A B^{4}}{S^{\prime 4}}\left(y-y_{0}\right) \dot{y}_{0} . \\
-\frac{48 A B^{4}}{S^{\prime 5}}\left(y-y_{0}\right)^{3} \dot{y}_{0}-\frac{8 A B^{6}}{S^{\prime 6}}\left(y-y_{0}\right)^{3} \dot{y}_{0}+\frac{48 A B^{4}}{S^{\prime 5}}\left(x-x_{0}\right)\left(y-y_{0}\right)^{2} \dot{x}_{0} . \\
-\frac{8 A B^{6}}{S^{\prime 6}}\left(x-x_{0}\right)\left(y-y_{0}\right)^{2} \dot{x}_{0}-\frac{8 A B^{2}}{S^{\prime 3}}\left(x-x_{0}\right) \dot{x}_{0}+\frac{4 A B^{4}}{S^{4}}\left(x-x_{0}\right) \dot{x}_{0} \\
-\frac{48 A B^{2}}{S^{\prime 4}}\left(x-x_{0}\right)\left(y-y_{0}\right)^{2} \dot{x}_{0} .
\end{gathered}
$$

We have used $\left(x-x_{0}\right)^{2}+\left(y-y_{0}\right)^{2}=S^{\prime}+R^{2}$ obtain the following

$$
\begin{gathered}
\psi_{t x x}+\psi_{t y y}+\beta \psi_{x}=\left\{\frac{24 A B^{2}}{S^{\prime 3}}\left[\left(x-x_{0}\right) \dot{x}_{0}+\left(y-y_{0}\right) \dot{y}_{0}\right]-\frac{48 A B^{2}\left(S^{\prime}+R^{2}\right)}{S^{\prime 4}}\left(x-x_{0}\right) \dot{x}_{0}\right. \\
-\frac{48 A B^{4}\left(S^{\prime}+R^{2}\right)}{S^{\prime 5}}\left(x-x_{0}\right) \dot{x_{0}}-\frac{48 A B^{4}\left(S^{\prime}+R^{2}\right)}{S^{\prime 5}}\left(y-y_{0}\right) \dot{x_{0}}+\frac{12 A B^{4}}{S^{\prime 4}}\left[\left(x-x_{0}\right) x_{0}+\left(y-y_{0}\right) \dot{y}_{0}\right] \\
-\frac{8 A B^{6}\left(S^{\prime}+R^{2}\right)}{S^{\prime 6}}\left(x-x_{0}\right) \dot{x}_{0}-\frac{8 A B^{62}}{S^{\prime 6}}\left(y-y_{0}\right) \dot{y}_{0}\left(S^{\prime}+R^{2}\right)-\frac{48 A B^{2}}{S^{\prime 4}}\left(y-y_{0}\right) \dot{y}_{0}\left(S^{\prime}+R^{2}\right) \\
\left.+\frac{8 A B^{2}}{S^{\prime 3}}\left[\left(x-x_{0}\right) \dot{x}_{0}+\left(y-y_{0}\right) \dot{y}_{0}\right]+\frac{4 A B^{4}}{S^{\prime 4}}\left[\left(x-x_{0}\right) \dot{x_{0}}+\left(y-y_{0}\right) \dot{y}_{0}\right]-\frac{2 A B^{2}}{S^{\prime 5}}\left(x-x_{0}\right)\right\} \\
=\left[\left(x-x_{0}\right) \dot{x}_{0}+\left(y-y_{0}\right) \dot{y_{0}}\right]\left\{\frac{24 A B^{2}}{S^{\prime 3}}-\frac{48 A B^{2}}{S^{\prime 4}}\left(S^{\prime}+R^{2}\right)-\frac{48 A B^{4}}{S^{\prime 5}}\left(S^{\prime}+R^{2}\right)\right. \\
\left.+\frac{12 A B^{4}}{S^{\prime 4}}-\frac{8 A B^{6}}{S^{\prime 6}}\left(S^{\prime}+R^{2}\right)+\frac{8 A B^{2}}{S^{\prime 3}}+\frac{4 A B^{4}}{S^{\prime 4}}\right\} \psi-\frac{2 A B^{2}}{S^{\prime 2}} \beta\left(x-x_{0}\right) \psi
\end{gathered}
$$

Assuming $\mathrm{R}=\mathrm{B}$ and putting $\left(x-x_{0}\right) \dot{x}_{0}+\left(y-y_{0}\right) \dot{y_{0}} \equiv \sum$ we get

$$
\begin{aligned}
& \psi t x x+\psi t y y+\beta \psi x=\sum\left\{\frac{A B^{2}}{S^{\prime 3}}(24-48+8)+\frac{A B^{4}}{S^{\prime 4}}(-48-48+12+4)+\frac{A B^{6}}{S^{\prime 5}}(-48-8)\right. \\
& \left.+\frac{A B^{8}}{S^{\prime 6}}(-8)\right\} \psi-\frac{2 A B^{2}}{S^{\prime 2}} \beta\left(x-x_{0}\right) \psi \\
& =\sum\left(-\frac{16 A B^{2}}{S^{\prime 3}}-\frac{80 A B^{4}}{S^{4}}-\frac{56 A B^{6}}{S^{\prime 5}}-\frac{8 B^{8} A}{S^{\prime 6}}\right) \psi-\frac{2 A B^{2}}{S^{\prime 2}} \beta\left(x-x_{0}\right) \psi .
\end{aligned}
$$

Recall that right hand side of the equation (4.1) is

$$
\left\{\widehat{G}_{x}-\frac{2 B^{2}}{S^{\prime 2}}\left(x-x_{0}\right) \hat{G}-\hat{F}_{y}+\frac{2 B^{2}}{S^{\prime 2}}\left(y-y_{0}\right) \hat{F}\right\} \psi
$$

If we now take $G=\beta$ the term in this expression arising from $\beta$ here cancels with the term proportional to $\beta$ on the 
right hand side of (4.5)

So we are left with

$$
G_{x}-\frac{2 B^{2}\left(x-x_{0}\right) \bar{G}}{S^{\prime 2}}+\frac{2 B^{2}\left(y-y_{0}\right) \hat{F}}{S^{\prime 2}}-\hat{F}_{y}=A B^{2}\left\{\frac{16}{S^{\prime 3}}+\frac{80 B^{2}}{S^{\prime 4}}+\frac{56 B^{\prime \prime}}{S^{\prime 5}}+\frac{8 B^{6}}{S^{\prime 6}}\right\} \sum
$$

We assume

$$
\bar{G}=\dot{x}_{0}\left(\frac{g_{1}}{S^{\prime 2}}+\frac{g_{2}}{S^{\prime 3}}+\frac{g_{3}}{S^{\prime 4}}\right), \hat{F}=\dot{y}_{0}\left(\frac{f_{1}}{S^{\prime 2}}+\frac{f_{2}}{S^{\prime 3}}+\frac{f_{3}}{S^{\prime 4}}\right)
$$

Therefore

$$
\begin{aligned}
& \bar{G}_{x}=-2\left(x-x_{0}\right) \dot{x}_{0}\left(\frac{2 g_{1}}{S^{\prime 3}}+\frac{3 g_{2}}{S^{\prime 4}}+\frac{4 g_{3}}{S^{\prime 5}}\right) \\
& \hat{F}_{y}=-2\left(y-y_{0}\right) \dot{y}_{0}\left(\frac{2 f_{1}}{S^{\prime 3}}+\frac{3 f_{2}}{S^{\prime 4}}+\frac{4 f_{3}}{S^{\prime 5}}\right)
\end{aligned}
$$

We now assume, $f_{1}=-g_{1}, f_{2}=-g_{2}, f_{3}=-g_{3}$; $\quad$ then we get

$$
\begin{aligned}
& \bar{G}_{x}-\frac{2 B^{2}\left(x-x_{0}\right) \bar{G}}{S^{\prime 2}}+\frac{2 B^{2}\left(y-y_{0}\right) \hat{F}}{S^{\prime 2}}-\hat{F}_{y} \\
& =\left[-2\left(x-x_{0}\right) x_{0}-2(y-y) y_{0}\right]\left[\frac{2 g_{1}}{S^{\prime 3}}+\frac{3 g_{2}}{S^{\prime 4}}+\frac{4 g_{3}}{S^{\prime 5}}+B 2\left(\frac{g_{1}}{S^{\prime 4}}+\frac{g_{2}}{S^{\prime} 5}+\frac{g_{3}}{S^{\prime 6}}\right)\right]
\end{aligned}
$$

Thus (4. 6) is satisfied

$$
g_{1}=4 B^{2} A, g_{2}=12 B^{2} A, g_{3}=4 B^{6} A
$$

For these values cause the right hand side of (4.7) to be equal to the right hand side of (4.6). Thus $\Psi$ given by (4.2a, b) and $\hat{G}, \hat{F}$ given by $(4,6 \mathrm{a})$ constitute an exact solution of the Rossby Equation (4.1); this is a solution

$$
u=-\psi_{y}=2 A B^{2}\left(y-y_{0}\right) \psi / S^{\prime 2}, v=\psi_{x}=-2 A B^{2}\left(x-x_{0}\right) \psi / S^{\prime 2} .
$$

Using arguments which are familiar by now [5] we see that these vanish strictly outside surface $S=0$.They also vanishes respectively far $x_{0}=x_{0}(t), y_{0}=y_{0}(t)$, which displays somewhat unusual behavior. It is interesting that the solution is valid and exact, for the given $F$ and $G$, for arbitrary motion of the centre $\left(x_{0}(t), y_{0}(t)\right)$ of the circle within which the disturbance is completely confined. It is also noteworthy that the radius of the circle within which the storm is confined stays the same as the circle moves. It may be possible to build more realistic models along the lines of this solution.

However, as investigating [7], [8] points out, for the Indian Ocean the linear theory gives good results. The nonlinear terms would be important for the Somali current, causing an 'inertial overshoot'; this can be seen from numerical studies of nonlinear effects [2], [4], [10].

\section{Acknowledgment}

We are grateful to the J. N. Islam, Professor, Research Centre for Mathematical and Physical Science, University of Chittagong, Bangladesh for carry out this investigation

\section{References}

[1] Anderson, D.L.T., and Rowlands, P.B. 1979 'The Somali current response to the S. W. Monsoon: The relative importance of local and remote forcing', J. Mar. Res. 34, 395-417.

[2] Bryan, k. 1963 'A numerical investigation of a non-linear model of wind-driven ocean', J. Atmos. Sci., 20, 594-606.

[3] Davies, A. M. 1991 International Journal for Numerical 
Methods in fluids, 12, 101Gill, A.E 1982 Atmosphere Ocean Dynamics. Academic Press (U. S. A).

[4] Gill, A.E. 1982 Atmosphere-Ocean Dynamics, Academic Press, New York.

[5] Islam, J.N. and Rahman, M.Z. 1998 "On the Dynamic Response of an Ocean to wind stress in an Equatoria Region: Exact Solutions of the Rossby Equation" national conference on applicable mathematics-2001, India.

[6] James, I. D. 1990 Modelling Marine Systems, 2, 345.

[7] Lighthill, M.J. 1969a 'Unsteady wind-driven ocean currents', Quart. J. Met. Soc. 95, 675-688.

[8] Lighthill, M.J. 1969b 'Dynamic response of the Indian Ocean to the onset of Southwest Monsoon': Philos. Trans. R. Soc. London Ser. A 265, 45-92.

[9] Richtmyer, R.D. 1978 Principles of Advanced Mathematical Physics, Vol. 1 Springer-Verlag, New York, Heidelberg and Berlin.

[10] Veronis, G. 1966 'Wind-driven Ocean circulation - Part-2. Numerical solution of the non-linear problem'. Deep-Sea Res. 13, 31-55. 